\title{
Proximity-induced superconductivity in graphene
}

\author{
M. V. Feigel'man* M. A. Skvortsov, and K. S. Tikhonov \\ L. D. Landau Institute for Theoretical Physics, Moscow 119334, Russia and \\ Moscow Institute of Physics and Technology, Moscow 141700, Russia
}

(Dated: October 1, 2008)

\begin{abstract}
We propose a way of making graphene superconductive by putting on it small superconductive islands which cover a tiny fraction of graphene area. We show that the critical temperature, $T_{c}$, can reach several Kelvins at the experimentally accessible range of parameters. At low temperatures, $T \ll T_{c}$, and zero magnetic field, the density of states is characterized by a small gap $E_{g} \leq T_{c}$ resulting from the collective proximity effect. Transverse magnetic field $H_{g}(T) \propto E_{g}$ is expected to destroy the spectral gap driving graphene layer to a kind of a superconductive glass state. Melting of the glass state into a metal occurs at a higher field $H_{g 2}(T)$.

PACS numbers: 74.78.-w, 74.20.-z, 74.81.-g
\end{abstract}

Among numerous fascinating properties, graphene 1, 2] provides a unique possibility to study the phenomenon of proximity-induced superconductivity in very favorable conditions. Experimental studies of the Josephson current through graphene in standard wide planar SNS junctions [3] have shown that proximity effect in graphene is qualitatively similar to the one known for usual dirty metals. In this Letter, we show that even a tiny amount of graphene area covered by small superconductive islands (with good electric contact to graphene) can lead to a macroscopically superconductive state of the graphene film, with $T_{c}$ in the Kelvin range.

We consider a system of superconductive (SC) islands of radius $a$ (with the typical value of few tenths of nanometer) placed approximately uniformly on top of a graphene layer (with the typical distance between islands $b$ in the sub-micron range) shown in Fig. 1. We assume that $b$ is much larger than both $a$ and the graphene meanfree-path $l$. Moreover, present theory will be limited by the case $l \lesssim a$ when electron motion in graphene is diffusive at all relevant scales. We will not be particulary interested in phenomena in the vicinity of the graphene neutral point, assuming relatively large gate potentials $\left|V_{g}\right| \geq 10 \mathrm{~V}$, and carrier density $n \geq 10^{12} \mathrm{~cm}^{2}$. We assume graphene Fermi energy $E_{F} \gg \Delta_{0} \gg T_{c}$, where $\Delta_{0}$ is the island's superconductive gap. Graphene sheet can be either single- or few-layered: the only relevant features are (i) high diffusion constant $D \geq 10^{2} \mathrm{~cm}^{2} / \mathrm{s}$, and (ii) very low (in comparison with metals) electron density, which allows to combine moderate values of dimensionless conductance $g=\left(\hbar / e^{2} R_{\square}\right) \geq 3$ with high Thouless energy $E_{\mathrm{Th}}=\hbar D / b^{2}$. Not very large values of sheet conductance $g$ are practically favorable to avoid suppression of superconductivity in small SC islands due to the inverse proximity effect [4].

Below we treat graphene as a normal diffusive 2D metal within the standard approach based on the Usadel equation [5]; its applicability to diffusive graphene was proven in Ref. [6, 7]. The intrinsic Cooper channel interaction in graphene can be neglected due to its low DOS [8].

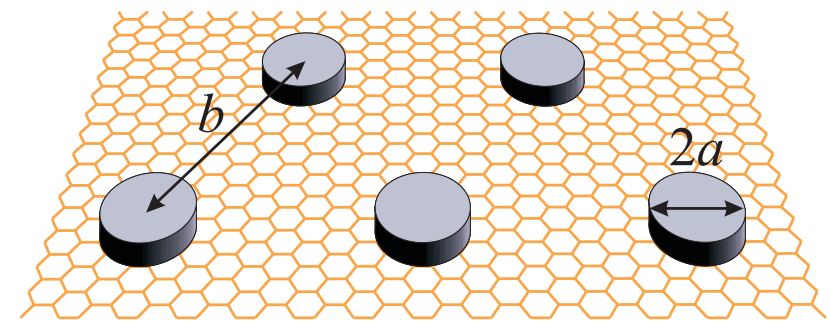

FIG. 1: (Color online) Graphene film covered by superconducting islands.

Similarly, phonon-induced attraction is also weak.

Proximity coupling and transition temperature.-We start with calculating the Josephson coupling energy between two superconductive islands of radius $a$ separated by distance $b \gg a$, neglecting the presence of other islands. Such a pair-wise approximation is adequate for determination of $T_{c}$, but breaks down at $T \leq T_{c} / \ln (b / a)$, as shown below. For the single SC island on graphene, the Matsubara-space Usadel equation for the spectral angle $\theta_{\omega}$ and corresponding boundary conditions $[9]$ read as

$$
\begin{gathered}
D \nabla^{2} \theta_{\omega}-2|\omega| \sin \theta_{\omega}=0, \\
{\left.\left[g \frac{\partial \theta_{\omega}}{\partial r}+\frac{G_{\text {int }}}{2 \pi a} \cos \theta_{\omega}\right]\right|_{r=a}=0 .}
\end{gathered}
$$

The normal $\left(G_{\omega}\right)$ and anomalous $\left(F_{\omega}\right)$ components of the matrix Green function the in Nambu-Gorkov space are expressed via the spectral angle $\theta_{\omega}$ and the order parameter phase $\varphi$ as $G_{\omega}(\mathbf{r})=\cos \theta_{\omega}(\mathbf{r})$ and $F_{\omega}(\mathbf{r})=$ $e^{i \varphi} \sin \theta_{\omega}(\mathbf{r})$. The full matrix structure of the anomalous Green function $\check{F}_{\omega}$ with the valley and spin spaces included (Pauli matrices $\hat{\pi}$ and $\hat{s}$, respectively) is determined by the usual $s$-wave pairing in the SC islands: $\check{F}_{\omega} \propto \hat{\pi}_{x} \hat{s}_{y}$. The interface conductance $G_{\text {int }}$ is treated below as a phenomenological parameter which accounts for Fermi velocity mismatch and a potential barrier on graphene-metal interface [10].

It is crucial for further analysis that the two-island generalization of the nonlinear problem (1), (2) can be lin- 
earized while calculating the Josephson current at interisland distances $b \gg a$. Indeed, the total current can be calculated by integrating the current density over the middle line between the islands, on the distance $\rho_{1,2} \geq b / 2$ from them. This procedure also involves summation over Matsubara energies $\omega_{n}=\pi T(2 n+1)$, with the major contribution to the sum coming from $\omega_{n} \sim E_{\mathrm{Th}}$. At such $\omega_{n}$ and $\rho_{1,2}$ the spectral angle $\theta$ is small, and linearization of Eqs. (11) and (2) leads to the solution

$$
\theta_{\omega}(r)=A(\omega) K_{0}\left(\frac{r}{L_{\omega}}\right), \quad A(\omega)=\frac{\Theta\left(t_{\omega}\right)}{\ln \left(L_{\omega} / a\right)},
$$

with $L_{\omega}=\sqrt{D / 2 \omega}, t_{\omega}=\left(G_{\text {int }} / 2 \pi g\right) \ln \left(L_{\omega} / a\right)$, and $\Theta(t)$ solving the equation $\Theta(t)=t \cos \Theta(t)$. The function $A(\omega)$ evolves between the tunnel and diffusive limits as

$$
A(\omega)= \begin{cases}G_{\mathrm{int}} /(2 \pi g), & G_{\mathrm{int}} \ll 2 \pi g / \ln \left(L_{\omega} / d\right), \\ \pi /\left[2 \ln \left(L_{\omega} / d\right)\right], & G_{\mathrm{int}} \gg 2 \pi g / \ln \left(L_{\omega} / d\right),\end{cases}
$$

and is always small for $\ln \left(L_{\omega} / a\right) \gg 1$. Thus the Josephson current $I(\varphi)=I_{c} \sin \varphi$ between two SC islands with different phases, $\varphi_{1}-\varphi_{2}=\varphi$, can be calculated using the linearized two-island solution for the anomalous Green function: $F_{\omega}(\mathbf{r})=e^{i \varphi_{1}} \sin \theta_{\omega}\left(\left|\mathbf{r}-\mathbf{r}_{1}\right|\right)+e^{i \varphi_{2}} \sin \theta_{\omega}(\mid \mathbf{r}-$ $\left.\mathbf{r}_{2} \mid\right)$. The standard calculation of the Josephson energy $E_{J}=(\hbar / 2 e) I_{c}$ then leads to

$$
E_{J}(b, T)=4 \pi g T \sum_{\omega_{n}>0} A^{2}\left(\omega_{n}\right) P\left(\sqrt{\omega_{n} / 8 E_{\mathrm{Th}}}\right),
$$

where $P(z)=z \int_{0}^{\infty} K_{0}(z \cosh t) K_{1}(z \cosh t) d t$.

A two-dimensional array of SC islands with the coupling energies (5) undergoes the Berezinsky-KosterlitzThouless transition at

$$
T_{c}=\gamma E_{J}\left(b, T_{c}\right),
$$

where the numerical coefficient $\gamma$ depends on the array structure. Below we will assume that the SC islands form a triangular lattice, in which case $\gamma \approx 1.47[11$. For the interface conductance $G_{\text {int }}$ comparable with the sheet conductance $g$, one finds the transition temperature $T_{c} \sim E_{\mathrm{Th}}$. In general, $T_{c}$ can be obtained by numerical solution of Eq. (6) using Eqs. (3) and (5). The result obtained for the ratio $T_{c} / E_{\mathrm{Th}}$ as a function of $G_{\text {int }}$ for $g=6$ $\left(R_{\square} \approx 700 \Omega\right)$ and $b / a=10$ is presented in Fig. 2. With the graphene diffusion constant $D=500 \mathrm{~cm}^{2} / \mathrm{s}$ (see, e.g., [12]) and $b=0.5 \mu \mathrm{m}$, one estimates $E_{\mathrm{Th}} \approx 1.5 \mathrm{~K}$, leading to $T_{c}$ in the range $1 \div 3 \mathrm{~K}$ for $5<G_{\text {int }}<20$.

Low temperatures: spectral gap and order parameter. Now we switch to the low-temperature range $T \ll T_{c}$ and consider the issue of the spectral gap for the excitation above the fully coherent ground state (with all phases $\varphi_{i}$ equal). The density of states $\nu(E)=\nu_{0} \operatorname{Re} \cos \theta(E)$ is determined then by the periodic solution of Eqs. (11) and (2),

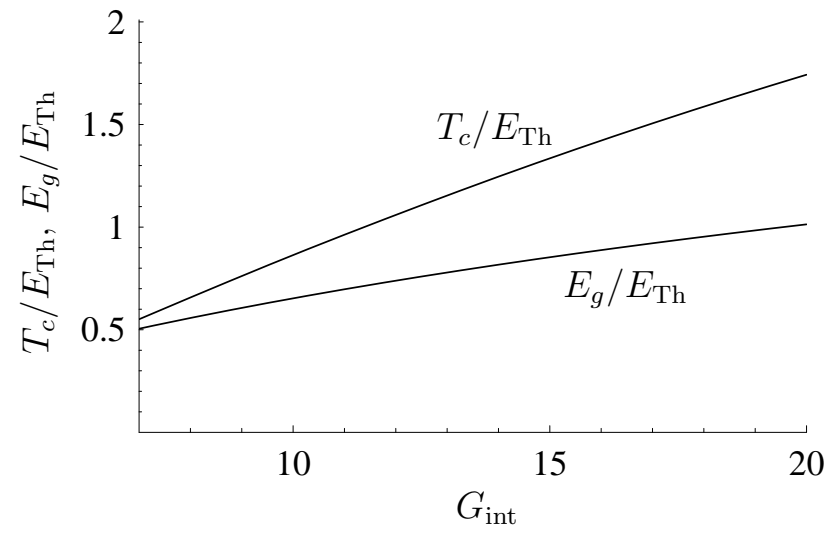

FIG. 2: The critical temperature, $T_{c}$, and the zerotemperature spectral gap, $E_{g}$, vs. the interface conductance $G_{\text {int }}$ (the sheet conductance $g=6$, and $b / a=10$ ).

analytically continued to real energies: $|\omega| \rightarrow i E$. This periodic problem is equivalent to the one defined within the single (hexagonal) elementary cell, supplemented by the additional condition $\left.\mathbf{n} \nabla \theta\right|_{\mathbf{r} \in \Gamma}=0$, where $\Gamma$ is the cell boundary. Solution of the Usadel equations for such a geometry leads to formation of the spectral gap $E_{g}$ similar to the minigap for one-dimensional SNS junctions [13]. To find it, we write $\theta(\mathbf{r})=\pi / 2+i \psi(\mathbf{r})$ and determine the spectral boundary as the value of $E$ where equation

$$
D \nabla^{2} \psi+2 E \cosh \psi=0
$$

ceases to have solutions with real $\psi(\mathbf{r})$ [14]. At large $\ln (b / a)$ one may approximate the hexagonal boundary $\Gamma$ of the elementary cell by the circle of radius $R=b / 2$. For the ideally transparent interface, numerical solution of the radially symmetric equation (7) gives for the value of the zero-temperature spectral gap:

$$
E_{g} \approx \frac{\hbar D / R^{2}}{1.52 \ln (R / a)-1.2} \approx \frac{2.6 E_{\mathrm{Th}}}{\ln (b / 4 a)} .
$$

Decreasing the interface conductance $G_{\text {int }}$ leads to the suppression of the the minigap, as shown in Fig. 2 ,

In the limit of large $\ln (b / a)$, the spectral gap $E_{g} \ll T_{c}$. Smallness of the gap distinguishes the system with superconductive islands from usual dirty superconductors. Roughly speaking, it behaves as a continuous 2D superconductor at the energy/temperature scales smaller than $E_{g}$, whereas in the range $E_{g}<(E, T)<T_{c}$ it can rather be described as an array of weak Josephson junctions.

The existence of the sharp gap (8) in the electron spectrum looks surprising, as only a tiny fraction $(a / b)^{2}$ of graphene area is in direct contact with $\mathrm{SC}$ islands. The presence of this gap can be traced back to the periodic structure of islands we assumed. Therefore any irregularity in the positions of SC islands will lead to the smearing of the hard gap. Assuming that islands' locations are shifted at random from the sites of the ideal triangular 
lattice, with the typical shift $\delta b \ll b$, one can reduce the problem to the effective one, defined on a scales large than array lattice constant. Random displacements of islands will be seen, in terms of this effective model, as local fluctuations of the superconductive coupling constant [15, 16], leading to the smearing of the gap with the relative width $\delta E_{g} \sim(\delta b / b)^{2} E_{g}$. The sharp gap will also be smeared by thermal fluctuations of island's phases $\varphi_{i}$ and finite thermal coherence length $L_{T}$. Thus we expect the spectral gap to be observable at $T \ll E_{g}$.

Even in the presence of the gap smearing, strong suppression of the local DoS in graphene at $E \leq E_{g}$, should be seen by the low-temperature Scanning Tunnelling Microscopy. The spectral (pseudo) gap is a signature of collective proximity effect which cannot be quantitatively described by a pair-wise interaction between SC islands as soon as low-energy scales $\leq E_{g}$ are involved. The corresponding spatial scale

$$
\xi_{g}=\sqrt{\hbar D / E_{g}} \sim b \sqrt{\ln (b / a)}
$$

plays the role of low-temperature coherence length in the (dirty-limit) superconductor. Under our main condition $\ln (b / a) \gg 1$, the coherence length $\xi_{g} \gg b$, which allows continuous treatment of the array at low temperatures.

The local superconductive order parameter in graphene, $\mathcal{F}(\mathbf{r})=\int d \omega F_{\omega}(\mathbf{r})$, can be found at $T<E_{g}$ as

$$
\mathcal{F}(\mathbf{r})=\sum_{j} \frac{D e^{i \varphi_{j}}}{\left(\mathbf{r}-\mathbf{r}_{j}\right)^{2}} \frac{\Theta\left[t\left(\left|\mathbf{r}-\mathbf{r}_{j}\right|\right)\right]}{\ln \left(\left|\mathbf{r}-\mathbf{r}_{j}\right| / a\right)}
$$

where $\mathbf{r}_{j}$ are the coordinates of SC islands, $t(r)=$ $\left(G_{\text {int }} / 2 \pi g\right) \ln (r / a)$, and we used the solution (3) for $\omega>E_{g}$. The divergent sum in Eq. (10) should be cut at $\left|\mathbf{r}-\mathbf{r}_{j}\right| \sim \xi_{g}$ since the spectral gap (8) suppresses the lowest- $\omega$ contribution to $\mathcal{F}(\mathbf{r})$. Equation (10) is not applicable in the vicinity of SC islands since Eqs. (11) and (2) cannot be linearized at small $\left|\mathbf{r}-\mathbf{r}_{j}\right|$.

At zero magnetic field, $\varphi_{j}=$ const and the spaceaveraged order parameter $\overline{\mathcal{F}}$ is given by

$$
\overline{\mathcal{F}}=n_{\mathrm{i}} \int_{b}^{\xi_{g}} d^{2} r \frac{D}{r^{2}} \frac{\Theta[t(r)]}{\ln (r / a)}=\frac{\pi^{2}}{2} n_{\mathrm{i}} D \frac{\ln \ln (b / a)}{\ln (b / a)},
$$

where the last expression refers to the large- $G_{\text {int }}$ limit $(\Theta \approx \pi / 2)$ and $n_{\mathrm{i}} \approx 1 / b^{2}$ is the concentration of SC islands. Comparison of (11) and (8) provides the condition for neglecting the intrinsic Cooper-channel interaction in graphene, $\lambda_{g}$. Namely, its presence would generate the the energy gap $\Delta_{g}=\lambda_{g} \overline{\mathcal{F}}$. This "intrinsic" gap can be neglected compared to proximity-induced gap (8) provided that $\lambda_{g} \ll 0.5$. Comparison with the estimate for intrinsic Cooper interaction constant [8] shows that the latter is indeed negligible.

Electromagnetic response. - Linear response of the superconductive film to a weak electromagnetic field is characterized by the superconductive density $\rho_{s}$. In the intermediate temperature range, $E_{g} \ll T \ll T_{c}$, one can easily calculate $\rho_{s}$ within the pair-wise approximation for the proximity coupling:

$$
\rho_{s}(T)=\frac{n_{i}^{2}}{2} \int_{0}^{\infty} 2 \pi r^{3} E_{J}(r, T) d r .
$$

Taking $E_{J}(r, T)$ from Eq. (5) we find

$$
\rho_{s}(T)=\left(\pi^{3} / 3\right) g A^{2}(\pi T) E_{\mathrm{Th}}^{2} / T,
$$

where $g=2 \nu_{0} D$ and the numerical factor corresponds to the triangular array with $n_{\mathrm{i}}=(2 / \sqrt{3}) b^{-2}$. At lowest temperatures, $T<E_{g}$, the function $\rho_{s}(T)$ saturates at the value that can be estimated by the replacement $T \rightarrow$ $E_{g}$ in Eq. (13). For highly transparent interface we obtain

$$
\rho_{s}(0) \approx 10 g E_{\mathrm{Th}} / \ln (b / a) .
$$

Comparing (14) and (8) we find that $\rho_{s}(0) \approx 4 g E_{g}$, which is typical for dirty superconductors with the gap $E_{g}$.

The critical current density per unit length at the lowest temperatures, $T \ll E_{g}$, can be estimated as

$$
j_{c}(0) \approx \frac{2 e}{\hbar b} E_{J}(b, 0)=\frac{\pi^{3}}{2} \frac{e g D}{b^{3} \ln ^{2}(b / a)},
$$

where we used, as an estimate, the $T=0$ limit of the pair-wise Josephson coupling energy (5) at $G_{\text {int }} / g \gg 1$ :

$$
E_{J}(b, 0)=\frac{\pi^{3}}{4} \frac{g E_{\mathrm{Th}}}{\ln ^{2}(b / a)} .
$$

The effect of the transverse magnetic field is characterized by two different field scales:

$$
H_{g}=\frac{\Phi_{0}}{2 \pi \xi_{g}^{2}} \approx \frac{0.4}{\ln (b / 4 a)} \frac{\Phi_{0}}{b^{2}}, \quad H_{\text {Glass }}=\frac{\Phi_{0}}{b^{2}} .
$$

In the low-field, low- $T$ region $\left(B \ll H_{g}\right.$ and $\left.T \ll E_{g}\right)$, magnetic field produces well-separated pancake "hypervortices" with the core size $\xi_{g} \geq b$ (the local DoS is gapless in the core regions). These vortices are strongly pinned by the underlying array structure, so a high critical current $j_{c 1} \sim j_{c}(0)\left(b / \xi_{g}\right)$ is expected. At $B \approx H_{g}$ vortex cores overlap and the proximity gap is totally destroyed, so $H_{g}$ is an analogue of the upper critical field $H_{c 2}$. However, the metallic state is not formed right above $H_{g}$, at least at $T \ll E_{g}$. In this field range one deals with a system of frustrated pair-wise Josephson couplings, with full frustration achieved at $B \gg$ $H_{\text {Glass. }}$. In this high-field range, average values of Josephson coupling are exponentially suppressed, $\overline{E_{J}(B)} \propto$ $\exp \left(-B / H_{\text {Glass }}\right)$. However, as shown in [17, 18], actual (random-sign) Josephson couplings are much stronger due to mesoscopic fluctuations:

$$
E_{J}^{\text {glass }}(b)=\left[\overline{\left(E_{J}(b)\right)^{2}}\right]^{1 / 2} \sim \frac{E_{\mathrm{Th}}}{\ln ^{2}(b / a)},
$$




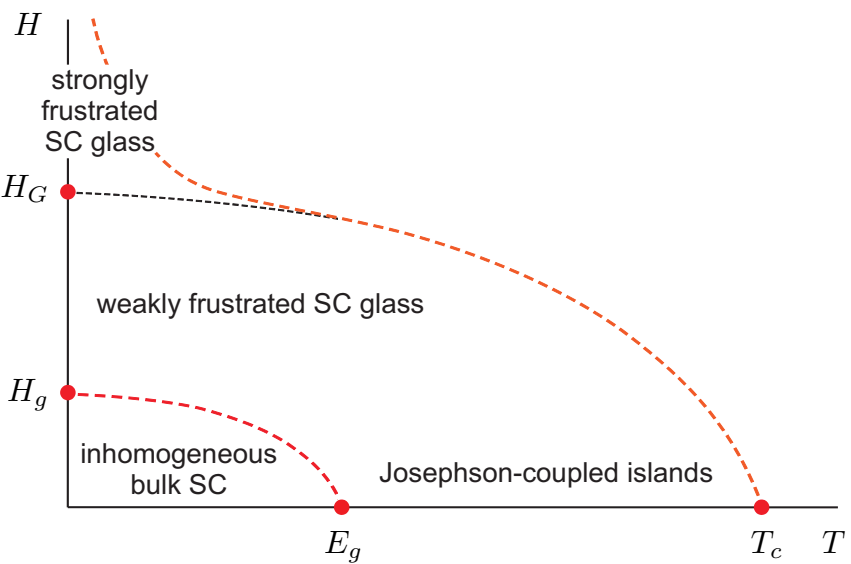

FIG. 3: Schematic phase diagram of the graphene sheet with superconductive islands; all lines refer to crossovers rather than to sharp phase transitions.

which is just by the factor $1 / \mathrm{g}$ smaller than the $(T, B)=0$ pairwise coupling (16). The estimate (18) shows that at $T \rightarrow 0$ the superconductive glass state survives up to high magnetic fields $H_{g 2}(0) \gg H_{\text {Glass }}$. The value of $H_{a 2}(0)$ is determined by quantum phase fluctuations [18, 19]:

$$
H_{g 2} \sim\left(\Phi_{0} / b^{2}\right) e^{c \sqrt{g}}, \quad c \sim 1 .
$$

The overall phase diagram in the $(H, T)$ plane is shown schematically in Fig. 3. We emphasize that the lines indicated do not refer to sharp phase transitions (which are absent in the presence of magnetic field, apart from some special values of frustration for the case of well-defined lattice of islands) but rather mark a crossover regions. Note that determination of $T_{c}$ for a rational values of frustration $f=\frac{1}{4}, \frac{1}{3}, \frac{1}{2}$ (where results for nearest-neighbor $\mathrm{XY}$ model are available) is complicated by the necessity of accounting for long-range proximity couplings.

To conclude, we have shown that graphene can be made superconductive with $T_{c}$ of the order of few Kelvins, due to collective proximity effect induced by small superconductive islands covering only a tiny part of graphene sheet area. The spectral gap is expected at low temperatures $T \ll T_{c}$ and magnetic fields $B \ll \Phi_{0} / b^{2}$. Transformation from a continuous disordered superconductive state to a weakly coupled junction array is predicted with the temperature and/or magnetic field increase.

Our study was based on the standard Usadel equations which are valid for sufficiently disordered samples $(l \ll a)$. In the opposite limit of quasi-ballistic electron motion around islands, the Andreev subgap conductance might decrease, leading to the increase of quantum fluctuations of phases $\varphi_{i}(t)[19,20]$. Quantum fluctuations can be neglected under the condition $b^{2} \ln (b / a) \ll b_{c}^{2}$, where $b_{c}$ is the critical distance between the islands marking the quantum phase transition (QPT) to the metal- lic state [19]. The same problem of suppressed Andreev conductance appears to be even more serious with the decrease of the electron density towards the graphene neutral point: scattering cross-section of electrons on the $\mathrm{SC}$ islands drops in the range of $k_{F} a \sim 1$ leading to effective decoupling of island's phases and to strong quantum fluctuations. These fluctuations may lead to the QPT of the superconductor-metal type [19, 20].

We are grateful to P. A. Ioselevich, P. M. Ostrovsky and M. Titov for useful discussions. This work was partially supported by RFBR Grant No. 07-02-00310.

* Electronic address: feigel@landau.ac.ru

[1] K. Novoselov et al, Science 306, 666 (2004).

[2] A. H. Castro Neto et al, arXiv:0709.1163.

[3] H. Heersche et al, Nature 446, 56 (2007); Xu Du, I. Skachko, and E. Andrei, Phys. Rev. B 77, 184507 (2008).

[4] The inverse proximity effect can be neglected provided that $G_{\text {tot }} \delta \leq \Delta_{0}$, where $G_{\text {tot }}^{-1}=G_{\text {int }}^{-1}+\ln (b / a) / 2 \pi g \ll 1$ is the total normal-state resistance from the island to the graphene sheet, $G_{\text {int }}$ is the SC-graphene interface conductance, and $\delta$ is level spacing in the island.

[5] K. Usadel, Phys. Rev. Lett. 25, 507 (1970).

[6] M. Titov, unpublished.

[7] K. S. Tikhonov and S. V. Kopylov, unpublished.

[8] In graphene, repulsion in the Cooper channel involves electrons from different valleys (wavevectors near the $K$ and $K^{\prime}$ points), which means that the Coulomb scattering amplitude is $U_{C}=2 \pi e^{2} /\left(\kappa K_{0}\right)$, where $\kappa \approx 5$ is the effective dielectric constant of graphene on the substrate and $K_{0}=1.7 \cdot 10^{8} \mathrm{~cm}^{-1}$ is the distance between $K$ and $K^{\prime}$ points in the reciprocal space. The dimensionless (repulsive) coupling constant is very small, $-\lambda_{g}=U_{C} \nu_{0}=$ $\left(2 e^{2} / \kappa \hbar v_{F}\right)\left(k_{F} / K_{0}\right) \leq 0.03$ for $n=k_{F}^{2} / \pi<10^{13} \mathrm{~cm}^{-2}$, due to the DOS $\nu_{0}=k_{F} / \pi \hbar v_{F}$ per single spin projection.

[9] M. Yu. Kupriyanov and V. F. Lukichev, Zh. Eksp. Teor. Fiz. 94, 139 (1987) [Sov. Phys. JETP 67, 1163 (1988)].

[10] B. Huard, N. Stander, J. A. Sulpizio, D. GoldhaberGordon, arXiv:0804.2040

[11] W. Y. Shih and D. Stroud, Phys. Rev. B 32, 158 (1985).

[12] F. V. Tikhonenko, D. W. Horsell, R. V. Gorbachev, and A. K. Savchenko Phys. Rev. Lett. 100, 056802 (2008)

[13] A. A. Golubov and M. Yu. Kupriyanov, Sov. Phys. JETP 69, 805 (1989).

[14] P. M. Ostrovsky, M. A. Skvortsov, M. V. Feigel'man, Phys. Rev. Lett. 87, 027002 (2001).

[15] A. I. Larkin and Yu. N. Ovchinnikov, Zh. Eksp. Teor. Fiz. 61, 2147 (1971) [Sov. Phys. JETP 34, 1144 (1972)].

[16] J. S. Meyer and B. D. Simons, Phys. Rev. B 64, 134516 (2001).

[17] B. Spivak and F. Zhou, Phys. Rev. Lett. 74, 2800 (1995).

[18] V. M. Galitskii and A. I. Larkin, Phys. Rev. Lett. 87, 087001 (2001).

[19] M. V. Feigel'man, A. I. Larkin, and M. A. Skvortsov, Phys. Rev. Lett. 86, 1869 (2001).

[20] M. V. Feigel'man and A. I. Larkin, Chem. Phys. 235, 107 (1998). 